\title{
El Faktum de la razón como actividad autoconstitutiva. Sobre la fundamentación de la moralidad kantiana
}

\author{
[The Fact of Reason as Self-Constitutive Activity. On the Foundation \\ of the Kantian Morality]
}

\author{
Gustavo Macedo Rodríguez \\ Carl von Ossietzky Universität Oldenburg \\ gmacedor@gmail.com
}

\begin{abstract}
Resumen: En el § 7 de la Crítica de la razón práctica Kant expone su primera definición de la ley moral universal (a la cual llama "imperativo categórico"). Ahí afirma que la conciencia de ella es un "Faktum de la razón". La ambigüedad de esta expresión ha hecho que algunos autores argumenten que Kant no deduce la ley moral satisfactoriamente ni aclara cómo llegamos a ser conscientes de ella. Sin embargo estos críticos olvidan en sus análisis un aspecto relevante de la filosofía moral kantiana y que es de gran relevancia en los estudios contemporáneos de la filosofía de la acción: el carácter autoconstitutivo del yo o del sujeto racional y su papel en la fundamentación de la moralidad. Sostengo que el Faktum de la razón debe entenderse fundamentalmente como un acto autoconstitutivo.
\end{abstract}

Palabras clave: racionalidad, autoconstitución, ley moral, razón práctica, intuicionismo

\begin{abstract}
In $\S 7$ of the Critique of Practical Reason Kant presents his first definition of the universal law of morality - called the Categorical Imperative. In the same place, he affirms that being conscious of the law of morality is a Faktum of reason. The ambiguity of this statement has prompted some authors to argue that Kant does not deduce the moral law satisfactorily nor does he clarify how we become conscious of it. Nevertheless, they all forget in their analysis a relevant aspect of Kantian moral philosophy that turns out to be of great importance in contemporary studies of the philosophy of action - the self-constituting character of the rational subject and the role it plays within the foundation of morality. I claim that the Faktum of reason must be understood fundamentally as a self-constituting act.
\end{abstract}

Key words: rationality, self-constitution, moral law, practical reason, intuitionism 
hoc volo, sic iubeo, sit pro ratione voluntas.

Juvenal, Satura VI, 223

\section{Introducción}

Kant escribe en el apéndice de la Crítica de la razón práctica (Kritik der praktischen Vernunft; en adelante, $K p V$ ) que hay dos cosas que lo colman de admiración (Bewunderung) y respeto (Ehrfurcht): "El cielo estrellado sobre mí y la ley moral en mí". ${ }^{1}$ Estos dos "objetos" de conocimiento corresponden a la ya conocida distinción entre el mundo sensible y la moralidad (o mundo inteligible) en la filosofía kantiana. Con el primer objeto, el mundo sensible, se aborda el tema de las condiciones de posibilidad del conocimiento y su estructura cognitiva dentro de los límites de la razón. Esta tarea se examina con amplitud en su filosofía teórica y en especial en la Crítica de la razón pura (Kritik der reinen Vernunft; en adelante, $\mathrm{KrV}$ ) y los Prolegomena. La estrategia kantiana consiste en analizar la estructura de las representaciones que los seres humanos producen de los objetos externos (fenómenos). El otro objeto, la moralidad, no es resultado de la experiencia empírica ni su validez depende de ella, sino de una racionalidad a priori y libre. ${ }^{2}$ Por ello, una de las tareas centrales en obras como la $K p V$ y la Fundamentación de la metafísica de las costumbres (Grundlegung zur Metaphysik der Sitten (en adelante, GMS) consiste en establecer una ley moral válida para todo sujeto racional.

En lo que sigue analizo cómo llegamos a ser conscientes de esta ley moral. De acuerdo con Kant, la conciencia de la ley moral es un "Faktum de la razón". Algunas interpretaciones contemporáneas de esta expresión coinciden en afirmar que es oscura y, por ello, formulan posibles vías para aclararla en el marco de una teoría general de la ley moral. $^{3}$ Una de esas propuestas, introducida por Dieter Schönecker, con-

${ }^{1}$ Kant, $K p V$, A 288.

${ }^{2}$ En palabras de Kant: "Das zweite [das moralische Gesetz] fängt von meinem unsichtbaren Selbst, meiner Persönlichkeit an und stellt mich in einer Welt dar, die wahre Unendlichkeit hat, aber nur dem Verstände spürbar ist, und mit welcher (dadurch aber auch zugleich mit allen jenen sichtbaren Welten) ich mich nicht wie dort in bloß zufälliger, sondern allgemeiner und notwendiger Verknüpfung erkenne" (Apéndice de la $K p V, 300$ ). Tanto la $K p V$ como la GMS se citarán de acuerdo con la versión de la Academia Alemana. Todas las traducciones del inglés y del alemán en este texto son de mi autoría.

${ }^{3}$ Entre los principales autores que sostienen esta tesis podemos mencionar a Dieter Henrich, Dieter Schönecker, Klaus Steiglieder y Michael Wolff.

Diánoia, vol. LXIII, no. 80 (mayo de 2018). 
siste en reconstruir dicha deducción mediante la noción de sentimiento o de respeto. ${ }^{4}$ Schönecker sostiene que el Faktum de la razón es un tipo de experiencia moral acompañada por el sentimiento de respeto. Por otro lado, otros autores como Michael Wolff, Pauline Kleingeld y Marcus Willaschek intentan rescatar de manera indirecta el carácter autoconstititutivo de ciertos actos humanos que sugiere la expresión "Faktum de la razón". Los dos últimos (Kleingeld y Willaschek) subrayan el hecho de que el término Faktum se acerca más a la acepción del término alemán Tat que a la de Tatsache, ${ }^{5}$ ya que Tat implica un carácter activo y no sólo un hecho concreto realizado. ${ }^{6}$ Si bien estos autores muestran las tensiones o debilidades de la argumentación kantiana y, en los casos de Willaschek, Wolff y Kleingeld aportan elementos que apoyan la idea de una razón activa, ninguno de ellos acentúa la importancia central del carácter autoconstitutivo del yo, a pesar de que el mismo Kant ejemplifica su tesis del Faktum de la razón con el uso de la expresión latina "sic volo, sic iubeo".

En lo que sigue analizaré el concepto de Faktum en el contexto de la filosofía moral kantiana. En contra de la interpretación intuicionista de Schönecker y de la crítica de Henrich, y retomando el carácter activo de la razón implícito en la expresión latina ya mencionada, mostraré que la teoría del Faktum de la razón implica en lo fundamental una teoría de la autoconstitución del yo. En otros términos, sosendré que el Faktum de la razón no es otra cosa que la unidad de la razón práctica, entendida como la relación analítica entre la actividad y su resultado.

\section{El Faktum de la razón como interpretamentum del imperativo categórico}

En el prefacio de la GMS Kant declara que su propósito central es "establecer un principio supremo de la moralidad". ${ }^{7}$ Este principio es el imperativo categórico y representa uno de los elementos centrales del

${ }^{4}$ Analizaré y refutaré esta tesis en la sección 2.

${ }^{5}$ El término alemán Faktum se puede traducir de las dos maneras, situación que hace más compleja su interpretación.

${ }^{6}$ Cfr. Willaschek 1992 y Kleingeld 2010. Para una posición contraria, aunque menos prometedora, véase Ware 2014, donde se sostiene que el Faktum de la razón es una teoría que debe entenderse como un experimento mental. La tesis de Ware consiste en afirmar que la filosofía moral responde más a una visión de los hechos desde la perspectiva de la primera persona y desde una perspectiva que llama "experimental".

${ }^{7}$ Kant, GMS, 392. 
programa de la filosofía práctica de Kant. Aclarar la función del imperativo categórico es decisivo si se pretende comprender la estructura interna de la filosofía moral kantiana. En este sentido, hay dos preguntas que deben responderse: ¿cómo es posible formular una ley moral universal válida para todo ser racional? Y ¿cómo puede un ser racional llegar a ser consciente de esa ley?, es decir, ¿bajo qué condiciones podemos formular esta ley? Y ¿cómo llegamos a tener conciencia de ella? Antes de abordar estas preguntas, debemos aceptar primero que del hecho de que pueda pensarse una ley moral universal no se sigue su validez. ${ }^{8}$ Más aún, es necesario explicar qué tipo de relación existe entre la formulación de la ley moral y su conciencia. ${ }^{9}$

Uno de los pasajes centrales en los que Kant intenta ofrecer una respuesta satisfactoria a las preguntas anteriores y fundamentar la relación entre la ley moral y su conciencia está en el $\S 7$ de la $K p V$. Ahí, Kant escribe lo siguiente:

Se puede llamar a la conciencia de esta ley fundamental un hecho de la razón porque no se puede deducir de datos anteriores de la razón, por ejemplo de la conciencia de la libertad (porque ésta no está dada a nosotros con anterioridad), sino porque la conciencia se nos impone como proposición sintética a priori, la cual no puede estar fundada ni en una intuición pura ni en una empírica. ${ }^{10}$

${ }^{8}$ Klaus Steigleder señala de manera oportuna este aspecto en Steigleder 2002, pp. 59 y ss.

${ }^{9} \mathrm{La}$ pregunta por la coherencia teórica entre la $G M S$ y la $K p V$, relevante en las discusiones actuales, no se abordará en este artículo. Mi interés principal es responder la pregunta de cómo llegamos a ser concientes de la ley moral y discutir las posibles respuestas en la $K p V$, donde justamente Kant introduce el término Faktum. A partir del análisis de la estructura argumentativa de Kant, algunos autores como Allen Wood han señalado que no es posible hablar de ruptura entre las dos obras, sino de una complementariedad, ya que Kant no desarrolla en la $K p V$, como sí lo hace en la GMS, una argumentación clara de la ley moral y su conciencia. Se trataría, más bien, de una nueva forma de hablar de la ley moral. Cfr. Wood 2008, pp. 134 y ss.

${ }^{10}$ Según Kant, "Man kann das Bewußtsein dieses Grundgesetzes ein Faktum der Vernunft nennen, weil man es nicht aus vorhergehenden Datis der Vernunft, z.B. dem Bewußtsein der Freiheit (denn dieses ist in uns nichts vorher gegeben) herausvernünfteln kann, sondern weil es sich für sich selbst uns aufdringt als synthetischer Satz a priori, der auf keiner, weder reinen noch empirischen, Anschauung gegründet ist, ob er gleich analytisch sein würde, wenn man die Freiheit des Willens voraussetzte, wozu aber, als positivem Begriffe, eine intellektuelle Anschauung erfordert werden würde, die man hier gar nicht annehmen darf". Kant, $K p V$, 141-142.

Diánoia, vol. LXIII, no. 80 (mayo de 2018). 
En este pasaje Kant afirma que la clave para entender la postulación del imperativo categórico y, por ende, la del establecimiento de una ley moral suprema, consiste en concebir la conciencia de dicha ley moral como un hecho de la razón (Faktum der Vernunft). Kant sostiene que la conciencia de la ley moral, que presupone la libertad de la voluntad, ${ }^{11}$ es el resultado de un "hecho (Faktum) de la razón", y advierte que la conciencia de la ley moral se nos impone como una proposición sintética a priori, es decir, que no proviene ni de una intuición pura ni de una empírica.

¿Qué es pues un "Faktum de la razón" y por qué no debe entenderse ni como resultado de una intuición pura ni de una empírica? Si seguimos a Kant, estas preguntas podrían responderse de la siguiente manera: el Faktum de la razón se relaciona de manera intrínseca con la actividad del sujeto. No se trata de ninguna intuición empírica o pura, porque el sujeto racional es consciente en forma inmediata tanto de la ley como del resultado de su propia actividad. Kant mismo hace explícita esta idea de la siguiente manera: "[la ley moral] no es algo empírico, sino que es el único Faktum de la razón pura, el cual es dado a conocer como originariamente dado (sic volo, sic iubeo)". ${ }^{12}$ Kant afirma en este pasaje que la ley moral es el único Faktum de la razón pura y que debe entenderse como algo dado. Sin embargo, esta tesis es ambigua. El Faktum de la razón podría ser resultado de la acción moral, pero también, al mismo tiempo, podría describir una actividad.

En Willascheck 1992 se traza una distinción importante en relación con la ambigüedad del concepto de Faktum en la filosofía moral kantiana. El autor sostiene que Kant usa el término en dos sentidos: "no sólo hace referencia a la actividad, sino al resultado o suceso, al hecho mismo"; es decir, el establecimiento de la ley moral y su conciencia son resultado de la racionalidad práctica. El Faktum de la razón puede referirse a Tat - el acto realizado por un sujeto- o bien a Tatsache, un hecho o resultado de una actividad. En este sentido, el Faktum es resultado de una racionalidad práctica, ya que el sujeto se hace consciente de la ley moral a partir de su actividad. ${ }^{13}$

Además, si retomamos el dictum "sic volo, sic iubeo", olvidado en los estudios especializados, podemos afirmar que el establecimiento de la ley moral y su conciencia configuran un proceso racional práctico que

${ }^{11} \mathrm{Cfr}$. Kant, $K p V, 142$.

${ }^{12}$ Kant, $K p V$, 142. El texto original es el siguiente: "daß es [Gesetz] kein empirisches, sondern das einzige Faktum der reinen Vernunft sei, die sich dadurch als ursprünglich gesetzgebend (sic volo, sic iubeo) ankündigt".

${ }^{13}$ Cfr. Willascheck 1992, pp. 180-181.

Diánoia, vol. LXIII, no. 80 (mayo de 2018). 
involucra tanto la actividad como su resultado. Como sabemos, el dictum "sic volo, sic iubeo", que podría traducirse como "así lo quiero, así lo ordeno" no es una expresión kantiana. ${ }^{14}$ Este dictum, que Kant toma y reinterpreta de Juvenal, puede ayudarnos a entender el razonamiento práctico que nos lleva a la conciencia de la ley moral y su establecimiento. Voluntad, mandato y ejecución son elementos de una racionalidad que implica una relación analítica entre la actividad y su resultado.

Las implicaciones de esta tesis que unifica el mandato racional y su realización a través de un acto revelan el carácter autoconstitutivo del establecimiento de la ley moral. Por "carácter autoconstitutivo" entiendo la función cognitiva de un ser racional de realizarse o actuar a partir de sus propias declaraciones y mandatos. En este caso, la acción incluye el proceso racional y su concretización; esto es, la relación entre la conciencia de la ley y su realización son elementos constitutivos de la acción. ${ }^{15}$ Por ello, se trata no sólo de simples declaraciones o deseos, sino de elementos fundamentales para la autodefinición y autoconstitución del sujeto. Con ello no se pretende argumentar que querer algo y realizarlo sean idénticos. Más aún, de acuerdo con la expresión reinterpretada por Kant "sic volo, sic iubeo", se trata de mostrar la conexión entre una acción sometida a la ley moral y su realización en el marco de una actividad autoconstitutiva. ${ }^{16}$

${ }^{14}$ La expresión "hoc volo, sic iubeo" remite a una de las sátiras más conocidas del poeta Juvenal, en la que intenta persuadir a un amigo de no casarse utilizando varios ejemplos de actos reprobables de mujeres de la época. La intención original de Juvenal era describir formas femeninas irracionales e injustificadas de actuar, producto de caprichos personales. Kant parte de esta relación entre querer y actuar para proponer una relación moral performativa. Véase Juvenal, Sátiras, VI, 223 (se analizará esta frase en detalle en la sección 3).

${ }^{15}$ Esta característica de la racionalidad práctica podría formularse también en términos del concepto moderno de performatividad, que consiste en la realización de declaraciones o enunciados que emite un sujeto. Expresiones como "Acepto" -en el contexto de una ceremonia religiosa - o "Nombro a este barco reina Elizabeth" - mientras que se rompe una botella contra el casco del barco que se pretende bautizar - son actos performativos porque dan lugar a hechos y acciones a partir de declaraciones. Cfr. Austin 1962 y Searle 1979. En el caso de Kant debemos agregar a esta definición que con estos actos el sujeto racional no sólo enuncia y realiza acciones, sino que se somete a una ley moral universal.

${ }^{16}$ Esta expresión se interpreta aquí como una relación analítica entre la conciencia y el resultado de una acción concorde con ella. Sin embargo, esta frase puede entenderse como la simple relación entre querer algo y realizarlo, que no siempre es analítica. Puedo querer algo y no necesariamente realizarlo. El matiz kantiano es claro: mis actos o acciones sometidos por la razón a la ley moral universal implican analíticamente la unidad entre la acción y el resultado.

Diánoia, vol. LXIII, no. 80 (mayo de 2018). 
Este carácter autoconstitutivo se relaciona con otro de los aspectos centrales y polémicos en la argumentación kantiana. Me refiero a la circularidad de la argumentación kantiana, la cual acepta Kant cuando afirma que toda voluntad libre presupone una voluntad sometida a la ley moral y viceversa. En otros términos, no es posible actuar libremente si nuestra voluntad no es moral, ni actuar moralmente sin suponer nuestra libertad. Esta circularidad se conoce como la tesis de la analiticidad ${ }^{17}$ y conecta dos características que se atribuyen al sujeto racional moral y que no pueden entenderse por separado. Al respecto, Kant escribe:

Nos aceptamos en el orden de las causas efectivas como libres para pensarnos en el orden de los fines bajo leyes morales, y nos pensamos, por lo tanto, como sometidos bajo la ley, porque nos hemos atribuido la libertad de la voluntad, porque la libertad y la legislación propia son ambas autonomía y, con ello, conceptos intercambiables que no pueden usarse sin aclarar al otro y dar razón de él, sino en el mayor de los casos solamente en la intención lógica de traer representaciones distintas y aparentes de los mismos objetos en un único concepto (como distintas rupturas del mismo contenido sobre las expresiones más pequeñas). ${ }^{18}$

La tesis de la analiticidad describe de manera puntual la circularidad de la argumentación de la ley moral. La ley moral, nos dice Kant, presupone la libertad de la voluntad. Responder por qué nos pensamos bajo una ley moral implica, según Kant, que nosotros nos suponemos una voluntad libre. De esta manera, libertad y moralidad son conceptos relacionales que se implican y explican uno al otro. ${ }^{19}$ Dicho en otros términos, toda voluntad libre se rige por una ley moral y viceversa. ${ }^{20}$

${ }^{17}$ Cfr. Schönecker 2013.

${ }^{18}$ Cfr. Kant, GMS, 450. El texto original es el siguiente: "Wir nehmen uns in der Ordnung der wirkenden Ursachen als frei an, um uns in der Ordnung der Zwecke unter sittlichen Gesetzen zu denken, und wir denken uns nachher als diesen Gesetzen unterworfen, weil wir uns die Freiheit des Willens beigelegt haben, denn Freiheit und eigene Gesetzgebung des Willens sind beides Autonomie, mithin Wechselbegriffe, davon aber einer eben um deswillen nicht dazu gebraucht werden kann, um den anderen zu erklären und von ihm Grund anzugeben, sondern höchstens nur, um, in logischer Absicht, verschieden scheinende Vorstellungen von eben demselben Gegenstande auf einen einzigen Begriff (wie verschiedne Brüche gleiches Inhalts auf die kleinsten Ausdrücke) zu bringen."

${ }^{19}$ De acuerdo con Kant, "una voluntad libre y una voluntad sujeta a la ley moral son lo mismo" (ein freier Wille und ein Wille unter sittlichen Gesetzen sind einerlei). Cfr. GMS, 447e.

${ }^{20}$ GMS, 450. 
Este "concepto de racionalidad práctica", ${ }^{21}$ que describe a un sujeto que se realiza mediante acciones morales y libres, configura la unidad entre el sujeto racional y el sujeto práctico. Esta forma argumentativa está presente también en la discusión de la teoría del Faktum de la razón, porque muestra precisamente cómo es posible entender la estructura ambigua de la moralidad.

A continuación analizo en detalle la expresión "Faktum de la razón" para mostrar la tesis de que el establecimiento de la ley moral universal y su conciencia es compatible con la tesis de una actividad autoconstitutiva.

\section{2. ¿Qué es un Faktum de la razón?}

¿Cómo entender la afirmación kantiana de que la conciencia de la ley moral, la cual es un Faktum de la razón, es algo dado al sujeto y cómo el sujeto racional hace comprensible la relación entre querer y deber? En concreto, ¿es el Faktum la condición de posibilidad de la acción entendida como una unidad entre la conciencia y la realización de la acción bajo la ley moral?

La última pregunta exige hacer primero una reflexión. La unidad entre la conciencia de la ley y la realización del acto nos conduce de manera irremediable a una ambigüedad: podemos hablar de una unidad dentro de la ley moral; es decir que sólo mi voluntad libre puede llegar a erigirse como acto moral y viceversa, o bien sólo comprometernos con una unidad práctica resultado de una acción racional. En este último caso sólo aceptaríamos una fórmula básica de la acción que, si bien afirma aún la unidad entre la actividad y su producto, no se compromete necesariamente con la moralidad de nuestros actos. En otros términos y para retomar una de las tesis de Wolff, se trata de discutir la distinción entre la ley moral y la ley práctica. ${ }^{22}$ El argumento de Wolff consiste en esencia en mostrar que hay una estructura básica que describe una unidad entre la conciencia y el resultado de la ley moral (la ley práctica). La ley práctica es resultado de la razón y describe un estado de cosas surgido de la autonomía de la voluntad. Esta ley debe distinguirse de la ley moral, la cual sería equiparable al imperativo categórico. Según Wolff, la distinción entre la ley moral y la ley práctica en el $\S 7$ de la $K p V$ hace comprensible la introducción de la teoría del Faktum de la razón. Ésta debería entenderse no como

${ }^{21}$ Cfr. Rivera 2014, p. 102.

${ }^{22}$ Cfr. Wolff 2009, pp. 524 y ss.

Diánoia, vol. LXIII, no. 80 (mayo de 2018). 
algo evidente para el sujeto racional, sino como algo dado (gegeben) ${ }^{23}$ es decir, Wolff introduce un carácter activo del Faktum de la razón y lo relaciona de manera directa con la ley práctica y no con la ley moral. Se trata, en pocas palabras, "de un postulado práctico que, de la misma manera que los postulados geométricos, goza de certeza apodíctica". ${ }^{24}$

Desde mi punto de vista, la tesis de Michael Wolff es acertada. El contenido de la ley moral y su conciencia forman una unidad constituida por el sujeto racional. Sin embargo, si se amplía la tesis de Wolff, antes de hablar de esta unidad debemos conceder que hay una unidad originaria o básica que se expresa a través de la capacidad de desear. $\mathrm{Si}$, como afirma Kant, esta ley moral y su conciencia no pueden ser producto de una intuición sensible ni de una pura, entonces debemos aceptar que se trata del resultado de una actividad autoconstitutiva. Pero esta tesis no refuta la posible deducción de la unidad de la ley moral y su conciencia. Más bien, acentúa la necesidad de reconstruir el proceso de realización de la conciencia de la ley moral bajo formas autoconstitutivas que anteceden a la ley moral. ${ }^{25}$ Los matices de esta interpretación van más allá del propio sistema de Kant y, en cierta medida, amplían perspectivas teóricas que no pueden discutirse aquí. ${ }^{26}$ La importancia de esta distinción radica en la necesidad de matizar la

${ }^{23}$ Wolff 2009, p. 526.

${ }^{24}$ En palabras de Wolff: “Die Rede vom 'Factum der Vernunft' hat nichts mit der Berufung auf eine Tatsache im Sinne einer Berufung auf Evidenz zu tun. Einer solchen Berufung bedarf es nicht, da das praktische Grundgesetz ein praktisches Postulat ist, dem als solchen (in derselben Weise wie geometrischen Postulaten) apodiktische Gewissheit zukommt. Um seine Gültigkeit einzusehen, genügt es zu wissen, was ein praktisches Gesetz ist und wovon seine Gültigkeit abhängt. Seine Gültigkeit hängt nicht (wie die Gültigkeit geometrischer Postulate) von einem Anschauungs- oder sonstigen Erkenntnisvermögen, sondern vom Begehrungsvermögen (dem Wollen) vernünftiger Wesen ab" (Wolff 2009, p. 549).

${ }^{25}$ Esta vía de argumentación no es nueva. El mismo Fichte, sobre todo en la época de Jena, acentuaba el carácter performativo y circular de la conciencia y su función básica que presupone todo acto, incluido el moral. Cfr. Fichte 1962 (1794-1795). Véase además Stolzenberg 1998. En las páginas siguientes analizaré detenidamente el concepto de performatividad.

${ }^{26}$ Las implicaciones de mi interpretación recuperan hasta cierto punto muchas de las críticas y reformulaciones de representantes del así llamado idealismo alemán. En particular, autores como Fichte y Schelling reprochaban a Kant la carencia de una teoría de la autoconciencia que unificara la razón páctica y la teórica a partir de un principio que funcionara como fundamento de toda acción humana. Fichte llamó a esta teoría "historia prágmática del espíritu humano" (Fichte 1962 [17941795]) y Schelling "historia de las autoconciencias" (Schelling 1985 [1800]). 
deducción kantiana a la luz de una forma básica de acción que haga comprensible la tesis del Faktum de la razón. Además, con la distinción que establece Wolff queda claro que la conciencia y la ley moral no son idénticas, sino partes constitutivas de la acción moral.

Según Wolff, Kant no ofrece tampoco una deducción en sentido estricto de la ley moral en la $K p V$ y supone su explicación en la GMS. Esto ha dado lugar a muchos malentendidos y a un callejón sin salida en la interpretación cuando pretendemos relacionar el Faktum con la ley moral o con el imperativo categórico. Tal es el caso de la propuesta interpretativa de Schönecker 2013, donde se afirma que la teoría del Faktum de la razón debe entenderse como un intuicionismo moral; esto es, que la conciencia de la ley moral es resultado no sólo de la racionalidad práctica, sino también de un sentimiento. ${ }^{27}$ La idea central de Schönecker es que el sentimiento (insight), entendido como respeto, forma parte de la estructura racional que nos permite explicar la conciencia moral. Sostiene además tres tesis respecto del Faktum de la razón que podrían conformar un argumento general y las enuncia de la siguiente manera:

1. La teoría del Faktum explica nuestra intuición en conexión con la ley moral, en el sentido de que esta teoría nos ofrece una justificación.

2. La ley moral se da inmediatamente en nuestra conciencia de la ley moral, en su validez incondicional y relacional.

3. La validez incondicional del imperativo categórico se da en el sentimiento de respeto. ${ }^{28}$

Schönecker aclara que su objetivo no es argumentar que la ley moral sea válida por el hecho de que tengamos un sentimiento o una intuición, sino que el imperativo categórico puede reconocerse justo por el sentimiento de respeto. ${ }^{29}$ En este sentido, la primera tesis que propone Schönecker acentúa el papel del sentimiento o intuición moral en la justificación de la ley moral. Somos capaces de reconocer que hay una ley moral entendida como imperativo categórico precisamente porque tenemos respeto por la ley moral. El imperativo categórico sería el primer paso para entender la ley moral como válida absolutamente. Según Schönecker, esta tesis se deduce de la premisa kantiana de la explicación mutua entre la libertad y la ley moral, la cual se formula en una

${ }^{27}$ Cfr. Schönecker 2013.

${ }^{28}$ Schönecker 2013, p. 3.

${ }^{29}$ Schönecker 2013, p. 4. 
nota al pie de página en el prólogo de la $K p V .^{30}$ Schönecker interpreta la tesis de la conciencia de la ley moral bajo el presupuesto de una analiticidad en el actuar moral: la libertad es la ratio essendi de la ley moral y la ley moral es la ratio cognoscendi de nuestra creencia de que somos libres. Con base en varias citas de la $K p V$, Schönecker asegura que el Faktum de la razón configura una vía de justificación de la conciencia de la ley moral. Sin embargo, advierte que existen pasajes en la obra de Kant que niegan el carácter justificativo del Faktum de la razón. ${ }^{31}$

Una vez aclarado el carácter justificativo en la teoría del Faktum, Schönecker conecta esta idea con la necesidad de entender al Faktum de la razón como algo dado, es decir, como algo autoevidente. El análisis de Schönecker parte de la ambigüedad de la expresión "Faktum de la razón". Según él, esta frase puede interpretarse como un genitivus subjectivus o como un genitivus objectivus. ${ }^{32} \mathrm{Si}$ decimos que es subjectivus, reconocemos que la ley moral o el imperativo categórico es un producto de la razón. En este sentido, el Faktum es un acto de la razón. Pero si la realidad de la libertad se expresa a sí misma en la conciencia de la ley moral y, por lo tanto, como producto de la razón, entonces hablamos de un genitivus objectivus. De este modo, Schönecker colige que la conciencia de la ley moral es un Faktum que la razón se da a sí misma. "Los seres humanos —afirma el autor- forman máximas para sí mismos como principios de determinación de la voluntad." ${ }^{33}$ Por esta razón, la pregunta kantiana de cómo es posible la conciencia de la ley moral, debe entenderse, si seguimos a Schönecker, como una pregunta por la manera en la que la ley moral debe ser dada. ${ }^{34}$ Para Schönecker, la conciencia de la ley moral es un Faktum de la razón en el sentido en de que el sujeto tiene evidencia de ella; es decir, que el sujeto moral posee un sentimiento de respeto [das Gefühl einer Achtung]. ${ }^{35}$

No obstante, entender la ley moral como algo dado resulta ambiguo e impreciso. Schönecker no analiza el problema de la ambigüedad en la

${ }^{30}$ Kant, $K p V$, nota a pie de página, p. 4.

${ }^{31}$ Entre otros, Schönecker cita los siguientes pasajes: $K p V: 91,33$; 91, 25; 47, 28. Sin embargo, al tratar de ofrecer una visión general del Faktum, Schönecker entra en un callejón sin salida cuando exacerba la confrontación erudita de muchos de los pasajes en la obra completa de Kant. Si explicamos la teoría del Faktum de la razón a partir de la confrontación de pasajes, queda claro que no tendremos una perspectiva única y satisfactoria, sino un impasse teórico.

${ }^{32}$ Antes que Schönecker, Henrich 1973 y Willaschek 1992 señalaron esta ambigüedad.

${ }^{33}$ Cfr. Schönecker 2013, p. 19.

${ }^{34}$ Schönecker 2013, p. 21.

${ }^{35}$ Schönecker 2013, p. 22. 
teoría del Faktum de la razón a pesar de alcanzar una conclusión innovadora: la teoría del Faktum de la razón contiene una ambigüedad o incluso una contradicción interna. Como ya se mencionó, el tema central de esta investigación es este presupuesto dentro del sistema kantiano: que el sujeto se dé a sí mismo la ley moral y tome conciencia de ella no significa otra cosa que la intuye inmediatamente porque está dada. ${ }^{36}$ En otros términos, el Faktum de la razón contiene ya una estructura ambigua y un carácter autoconstitutivo. ${ }^{37}$

Con los elementos críticos e interpretativos reunidos hasta ahora, argumentaré a continuación que de la estructura de la moralidad que establece la teoría del Faktum de la razón se deriva una estructura autoconstitutiva que, sin dejar de ser ambigua, explica la relación entre la ley moral y su conciencia, justamente en la medida en que se acepte una estructura originaria autoconstitutiva.

\section{El Faktum de la razón como un acto de autoconstitución}

Para los fines que planteé al inicio hay un aspecto relevante en las conclusiones de Schönecker, Henrich y Wolff. La teoría del Faktum de la razón describe una unidad sui generis en la que no está claro si la conciencia de la ley moral es resultado de una facultad cognitiva o si es producto de la actividad. Ni Schönecker ni Henrich profundizan en la discusión de la naturaleza de esta unidad, a pesar de que esta naturaleza hace patente el carácter autoconstitutivo del sujeto y hace comprensible la teoría del Faktum de la razón. La conciencia de la ley moral y la ley moral misma forman una unidad contenida en la actividad del sujeto racional. En cierto sentido, esta idea la avizoraron Willaschek y Kleingeld, quienes acentúan esta ambigüedad y entienden el término Faktum como Tat y no como Tatsache. ${ }^{38}$ Sin embargo,

${ }^{36}$ Autores como Henrich, Schönecker y Willaschek apuntan además que hay una fuerte conexión entre esta autoevidencia y el sentimiento de respeto. Cfr. Henrich 1973, p. 249; Schönecker 2013 y Willaschek 1992, pp. 174-193.

${ }^{37}$ Antes de ser expuesta por Schönecker, la ambigüedad de la teoría del Faktum de la razón fue discutida décadas atrás por Dieter Henrich, quien caracteriza la teoría del Faktum de la razón como una contradicción, en su influyente artículo Heinrich 1973. Según este autor, si un sujeto es consciente de la ley moral porque es algo dado a la razón y si es algo autoevidente para el sujeto, entonces no queda claro si se trata de una facultad cognitiva a priori o un producto (hecho) empírico. Kant no nos explica, según la argumentación de Henrich, el carácter contradictorio de la teoría del Faktum de la razón. Cfr. Henrich 1973.

${ }^{38}$ Ésta es justo la ambigüedad que subraya Schönecker y que, de manera extraña, no aborda con profundidad en Schönecker 2013. 
ni Willaschek ni Kleingeld enfatizan la naturaleza autoconstitutiva que describe la teoría del Faktum de la razón, la cual se expresa en el dictum "sic volo, sic iubeo" y que el mismo Kant utiliza para explicar el Faktum de la razón en la $\mathrm{KpV}$. Esta frase extrañamente olvidada en todos los estudios sobre la teoría del Faktum no es sólo un addendum, sino la clave para entender el carácter autoconstitutivo del Faktum de la razón.

En su sátira VI, Juvenal ofrece algunas razones para no contraer matrimonio. Mediante varios ejemplos de la época, intenta convencer a un amigo de las vicisitudes y desventajas del matrimonio. Después de ofrecer sus ejemplos, Juvenal introduce el dictum al referirse a la crucifixión de un esclavo. Se trata de una crucifixión ordenada por una mujer que no tiene otra razón sino su propia voluntad o querer. Cuando se le pregunta por las razones de su decisión y se le advierte que su acción debe ser reflexiva porque la vida de un hombre está en juego, responde: "No ha hecho nada, de acuerdo, pero lo quiero, lo ordeno. Sirva como razón mi voluntad". ${ }^{39}$ La arbitrariedad de mandar bajo la convicción de una voluntad irracional no es un aspecto que Kant rescate en su argumentación. Más aún, parece que le interesa salvar la unidad entre la voluntad de realizar algo y la realización del acto. El acto es el resultado de una voluntad y la acción la lleva a cabo el sujeto porque éste así lo quiere; la voluntad de querer algo es aquí la principal razón para hacerlo. Sin duda, esta fórmula básica de entender una acción puede exponerse también en términos racionales o deliberativos. Si la voluntad hace posible la realización de una acción, entonces es posible que la voluntad sujeta a la ley moral posibilite la realización de una acción moral.

Hay, además, un aspecto importante en la expresión "sirva como razón mi voluntad" (sit pro ratione voluntas) que es de especial interés en mi argumentación. Si el querer es un querer racional sometido a la ley universal, entonces bastaría como razón para la realización de la acción. No obstante, si analizamos esta relación desde una concepción básica de la acción, podríamos decir que la voluntad de hacer o querer algo es la razón para hacerlo; es decir, que no se requiere de ninguna razón para poder realizar algo, sino que "sólo" basta mi voluntad. Si esta relación se contempla desde la perspectiva de la primera persona, entonces podemos establecer una unidad entre querer y realizar una acción. Esta función originaria y básica describe con exactitud la distinción que traza Wolff entre la ley moral y la ley práctica. Podemos

${ }^{39}$ Juvenal, Satura VI, 223. 
distinguir entre una acción realizada y una acción pensada o sometida a una máxima moral. Sin embargo, ¿qué pasa cuando este querer se vuelve racional y se hace consciente de la ley moral? Kant mismo enfatiza la respuesta con ayuda del imperativo categórico en la observación del § 7 de la $K p V$. Ahí, Kant señala lo siguiente:

La ley moral es para aquel ser racional, por lo tanto, un imperativo que ordena categóricamente, porque la ley es incondicionada: la relación de tal voluntad con esa ley es de dependencia, bajo el nombre de obligatoriedad, la cual significa una coacción, y por lo tanto obligación, hacia un actuar, ciertamente a partir de la simple razón y aquella ley objetiva, porque un arbitrio afectado patológicamente (aunque no determinado patológicamente y por lo tanto siempre libre) implica un deseo que se origina en causas subjetivas y por ello puede ser frecuentemente opuesto también al fundamento determinante objetivo, y por lo tanto requiere de una resistencia de la razón práctica como coacción moral, la cual puede nombrarse constricción interna, pero intelectual. ${ }^{40}$

En esta cita Kant muestra que la dependencia entre la voluntad y la ley moral es necesaria. Aquí el tema central no es cómo llegamos a tener conciencia de ella, sino la obligatoriedad que se impone al sujeto racional respecto de ella, es decir que estamos obligados o coaccionados a actuar conforme a ella. De esta manera, la coacción (Nötigung) que se nos impone debe entenderse como una obligación impuesta por el mismo sujeto. El cumplimiento de esta obligación sólo puede realizarlo una voluntad. En otros términos, la voluntad y la obligación de actuar conforme a la ley moral deben considerarse una unidad inseparable. No podemos actuar conforme a una ley moral sin voluntad. Esta cita y el dictum "sic volo, sic iubeo" confirman la tesis de que sólo es posible estar sometido a la ley moral si aceptamos el carácter autoconstitutivo del sujeto racional. Dicho de otro modo, toda acción moral y su conciencia son resultado de un acto que involucra una facultad autoconstitutiva del sujeto. De manera implícita, Kant acentúa la unidad entre el sujeto que realiza la acción y la conciencia de la ley moral. Ambos aspectos se explican con la figura de un sujeto que se autoconstituye y, al hacerlo, vuelve explícita la unidad entre estar sujeto a la ley y su voluntad de ejecutar tal acción. Por lo tanto, el Faktum de la razón es una función autoconstitutiva que unifica la voluntad, la acción y la conciencia de la ley moral. El Faktum de la razón describe así el carácter autoconstitutivo del sujeto a partir del cual se realiza la ley moral.

${ }^{40}$ Kant, KpV, 143.

Diánoia, vol. LXIII, no. 80 (mayo de 2018). 


\section{Conclusiones}

He defendido con especial interés la tesis de que la formulación kantiana del Faktum de la razón implica fundamentalmente una actividad autoconstitutiva del sujeto racional. Cuando Kant afirma que la conciencia de la ley moral debe entenderse como un Faktum de la razón e insiste en la obligatoriedad que la ley moral impone a la voluntad, avizora ya la idea de que el sujeto racional se constituye a partir de su propia actividad. A diferencia de la lectura intuicionista del Faktum de la razón, expuesta en la primera parte de este artículo, mostré que el Faktum de la razón es fundamentalmente una actividad autoconstitutiva del sujeto racional. Que la conciencia de la ley moral sea un Faktum de la razón implica que el sujeto que se halla sometido a la ley moral decide realizarse (a partir de sus actos) y ciertamente con la conciencia de que es él el sujeto que las realiza. La teoría kantiana del Faktum de la razón inaugura de este modo el marco conceptual de la actividad autoconstitutiva del sujeto. De ahí podemos colegir el acento en la perspectiva de la primera persona en la fundamentación de la filosofía moral, porque es a partir de la voluntad del sujeto que tiene conciencia de la ley moral como éste se autoconstituye a partir de sus actos.

Esta idea la han sugerido con acierto autores como Willaschek y Kleingeld. Para ellos, el término Faktum implica un acto (Tat) y no sólo un hecho (Tatsache). Willaschek acentúa esta ambigüedad para describir el Faktum de la razón sobre todo como un estado de cosas en el que el sujeto dispone de motivos para actuar moralmente. ${ }^{41}$ Kleingeld coincide también en este punto y matiza que el término Faktum en el $\S 7$ de la $K p V$ debe entenderse como un hecho (Tatsache), algo existente, pero sin dejar de considerar que la conciencia de la ley moral es resultado de una acción. ${ }^{42}$

A diferencia de estos dos autores, en esta investigación mostré que la teoría del Faktum describe una racionalidad práctica que se caracteriza por una unidad entre la conciencia y la realización de la ley moral. La frase "sic volo, sic iubeo", que de manera extraña no analiza ni retoma ninguno de los autores mencionados en este ensayo, expresa justo la unidad entre querer algo y la realización de ese algo. En particular, este dictum sugiere una estructura cognitiva del sujeto racional que explica cómo el sujeto racional puede autoconstituirse moralmente. Esta idea refuta la tesis de Schönecker que pretende reducir la tesis del Faktum de la razón a un intuicionismo moral.

${ }^{41}$ Cfr. Willaschek 1992, p. 188.

${ }^{42}$ Cfr. Kleingeld 2010, pp. 64 y ss. 
A pesar de que el mismo Kant no lleva a sus últimas consecuencias esta tesis, es posible entrever con ella una nueva perspectiva en su filosofía. Se trata de una perspectiva que acentúa el análisis de aspectos constitutivos en la estructura de la voluntad y que acepta la ambigüedad de la acción y la conciencia. Aceptar esta naturaleza de la acción moral no sólo abre nuevas líneas de investigación —que ya han sido explotadas por teorías contemporáneas de la acción y la subjetividad en nuestro siglo-,${ }^{43}$ sino que es clave en la controversia posterior entre Kant y los idealistas alemanes, en especial con Fichte. Además, es relevante porque reinventa la capacidad de autoconstitución del sujeto en términos de estructuras básicas y originarias de la subjetividad. Esta última tesis, atribuida históricamente a sistemas filosóficos como los de Fichte, Schelling y Hegel, hace patente una afinidad hasta ahora negada entre Kant y sus sucesores y de la cual es necesario ocuparse.

\section{BIBLIOGRAFÍA}

Austin, J.L., 1962, How to Do Things with Words, Oxford University Press, Londres.

Ameriks, K., 2002, Interpreting Kant's Critiques, Clarendon Press, Oxford.

Frankfurt, H.G., 1971, "Freedom of the Will and the Concept of a Person", The Journal Philosophy, vol. 68, no. 1, pp. 5-20.

—_, 2001, Freiheit und Selbstbestimmung. Ausgewählte Texte, ed. M. Betzler, Akademie, Berlín.

Fichte, J.G., 1962, Grundlage der gesammten Wissenschaften als Handschrift für seiner Zuhörer, ed. R. Lauth et al., Frommann-Holzboog, Stuttgart-Bad Cannstatt [1a. ed.: 1794-1795].

Henrich, D. 1966, "Fichtes ursprüngliche Einsicht", en D. Henrich y H. Wagner (comps.), Subjektivität und Metaphysik. Festschrift für Wolfgang Cramer, Vittorio Klostermann, Fráncfort del Meno, pp. 88-233;

_, 1973, "Der Begriff der sittlichen Einsicht und Kants Lehre vom Faktum der Vernunft", en G. Prauss (comp.), Kant. Deutung seiner Theorie von Erkennen und Handeln, Kiepenheuer und Witsch, Colonia, pp. 223-254.

Husserl, E., 1988, "Kritik der Kantischen Ethik", en Vorlesungen über Ethik und Wertlehre 1908-1914, ed. U. Melle, Kluwer Academic (Husserliana, 28), Dordrecht/Boston/Londres, pp. 402-418.

Kant, I., 1968a, Werke in zwölf Bänden,vols. III y IV, Kritik der reinen Vernunft, ed. W. Weischedel, Suhrkamp, Fráncfort del Meno [1a ed.: 1787].

${ }^{43}$ En la bibliografía contemporánea hay varios planteamientos cercanos a mi posición; a pesar de que no todos ellos se comprometen con una estructura interna de la voluntad, sí acentúan el carácter autoconstitutivo y reflexivo del sujeto moralracional. Véanse Korsgaard 2009, Frankfurt 2001, Frankfurt 1971, Nagel 1986 y Nagel 1997.

Diánoia, vol. LXIII, no. 80 (mayo de 2018). 
Kant, I., 1968b, Werke in zwölf Bänden, vol. VII, Kritik der praktischen Vernunft. Grudlegung zur Metaphysik der Sitten, ed. W. Weischedel, Suhrkamp, Fráncfort del Meno [1a. ed. de la KpV: 1788; 1a. ed. de la GMS: 1785].

Kleingeld, P., 2010, "Moral Consciousness and the 'Fact of Reason'", en A. Reath y J. Timmermann (comps.), Kant's Critique of Practical Reason. A Critical Guide, Cambridge University Press, Cambridge, pp. 55-72.

Korsgaard, Ch., 2009, Self-Constitution. Agency, Identity, and Integrity, Oxford University Press, Nueva York.

Lazos, E., 2014, Disonancias de la Crítica. Tres ensayos sobre la Crítica de la razón pura, Instituto de Investigaciones Filosóficas-UNAM, México.

Mittmann, J.-P., 1993, Das Prinzip der Gewißheit. Fichte und die Entwicklung des nachkantischen Grundsatzphilosophie, Athenäum Hain Hanstein, Bodenheim.

Nagel, Th., 1986, The View from Nowhere, Oxford University Press, Oxford.

—, 1997, The Last Word, Oxford University Press, Oxford.

Rivera, F., 2014, Virtud, felicidad y religión en la filosofía moral de Kant, Instituto de Investigaciones Filosóficas-UnAM, México.

Schelling, F.W.J., 1985, System des transzendentalen Idealismus, en Ausgewählte Schriften in sechs Bänden, vol. I, 1794-1800, Fráncfort del Meno, Suhrkamp [1a. ed.: 1800].

Schönecker D. y A.W. Wood, 2002, Kants Grundlegung zur Metaphysik der Sitten. Ein einführender Kommentar, Schöningh, Paderborn.

— 2013, "Kant's Moral Intuitionism: The Fact of Reason and Moral Predispositions", Kant Studien Online, pp. 1-38.

Searle, J.R., 1979, Expression and Meaning. Studies in the Theory of Speech Acts, Cambridge University Press, Nueva York.

Steigleder, K., 2002, Kants Moralphilosophie. Die Selbstbezüglichkeit reiner praktischer Vernunft, J.B. Metzler, Stuttgart.

Stolzenberg, J., 1998, "Reiner Wille. Ein Grundbegriff der Philosophie Fichtes", Revue Internationale de Philosophie, vol. 4, no. 206, pp. 617-639.

Ware, O., 2014, "Rethinking Kant's Fact of Reason", Philosophers' Imprint, vol. 14, no. 32, pp. 1-21.

Willaschek, M., 1992, Praktische Vernunft. Handlungstheorie und Moralbegründung bei Kant, Metzler, Stuttgart.

Wolff, M., 2009, "Warum das Faktum der Vernunft ein Faktum ist. Auflösung einiger Verständnisschwierigkeiten in Kants Grundlegung der Moral", Deutsche Zeitschrift für Philosophie, vol. 57, no. 4, pp. 511-549.

Wood, A.W., 2008, Kantian Ethics, Cambridge University Press, Cambridge.

Recibido el 20 de junio 2016; 1a. revisión el 4 de enero de 2017; 2a. revisión el 22 de julio de 2017; aceptado el 11 de agosto de 2017. 